\title{
A Data Mining Analysis to evaluate the additional workloads caused by welding distortions
}

\author{
Losseau N., Caprace J.D., Aracil Fernandez F., Rigo P.
}

ABSTRACT: This paper presents a way to minimize cost in shipbuilding industry by using the results of a data mining analysis aiming to improve the cost knowledge of the additional operations caused by welding distortions. This statistical analysis had the scope to establish assessment formulas of the supplementary workloads in function of scantlings and welding distortions. Those formulas can be useful to evaluate the profitability of new welding devices and can improve the research in the following domains: production simulation, cost assessment of ship hull, structure optimization, design for production, etc.

\section{Introduction}

Since several years, the big shipyards use more and more plates of small thickness to build up the stiffened panels in order to decrease the structural weight of ships. The major problem relating to the utilization of thin plates is the appearance of welding distortions that have to be eliminated for fabrication, esthetical and service reasons. The supplementary operations to counter the problems of misalignment and lack of flatness involve non negligible costs and it seems thus important to characterize their economical impact on the hull production in function of welding distortions.

We distinguish several supplementary operations involved to the welding distortions:

- The steel working operations during the fixing of girders and bulkheads on panels to constitute sections.

- $\quad$ The sections edges' straightening that consists in adjusting the edges of neighbour sections to constitute blocks.

- $\quad$ The desks straightening that permits to recover a certain flatness of decks by heating techniques (see Figure 1)

The idea used to establish relations between those supplementary workloads and the residual welding distortions was to lead a statistical analysis basing on the production data from a shipyard. This paper gathers the results of several analyses realised with the so called data mining technique. The first analysis has exploited workload data of 13 passengers' ships in order to establish a formula linking the scantling (geometrical characteristics of stiffened panels) to the straightening cost [hour $/ \mathrm{m}^{2}$ ]. The second analysis was led further to a measure campaign gathering the welding distortions of one cruise ship and permitted to estimate the residual deformations in function of scantling. The last data mining studies realised recently had exploited production data and measurement campaign data in order to generate relations between distortions and supplementary works of adjusting and straightening. 

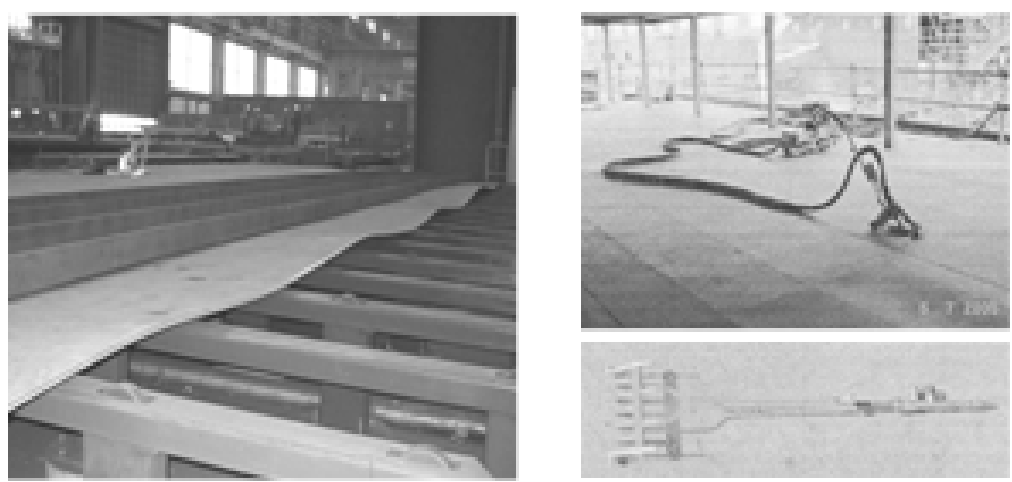

Figure 1 (a) A distorted panel.

Deck straightening techniques: (b) by induction, (c) with a 5 nozzles blowtorch.

\section{Decks straightening in function of scantling}

The first analysis aimed to generate a formula to estimate the decks straightening in function of scantling. This study was lead following the Data Mining methodology that is, by definition, the non-trivial process of extracting valid, previously unknown, comprehensible, and useful information from large databases. The successive steps of this data mining analysis are presented here after:

\subsection{Database creation}

A data base was first of all constituted; it gathered, for 13 passenger's ships, the characteristics of each section (global geometry, deck thickness, dimensions and inter-distance of stiffeners, section family, deck number, steel grade, section weight, etc.) and the associated straightening workload [hour].

\subsection{Data description stage}

This step consisted in a presentation of the attributes (fields of the data base), with their distribution and other statistical parameters (minimum, maximum, mean and variance). One of difficulties which arose during the database analysis is that the most structural attributes show a discrete distribution with one or few dominant modes (see Figure 3 (3) and (4)); for instance, the distance between stiffeners has very often the same value. Those attributes are almost "constant" parameters and thus don't constitute a conclusive information source. In order to minimize this effect, we have replaced some attributes. In this scope, we divided for example the plate weight by the section surface to obtain information similar to the thickness, but having the advantage to present a distribution much less discrete.

\subsection{Data quality stage}

This step listed the problematic recordings (strange distribution, missed values, data in conflict with their physical meaning) in order to take care of them in the next stages. 


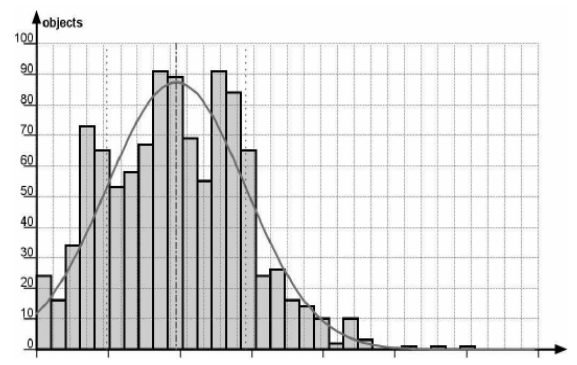

(1) Sections Surface

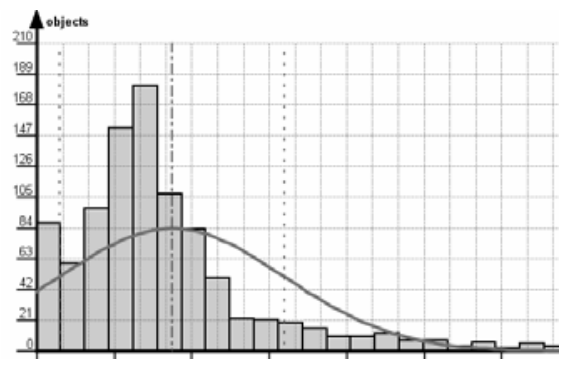

(2) Straightening time

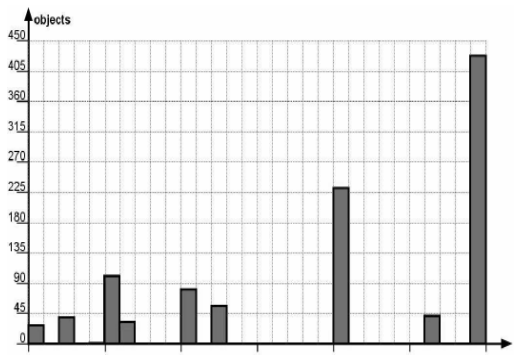

(3) Distrance between stiffeners

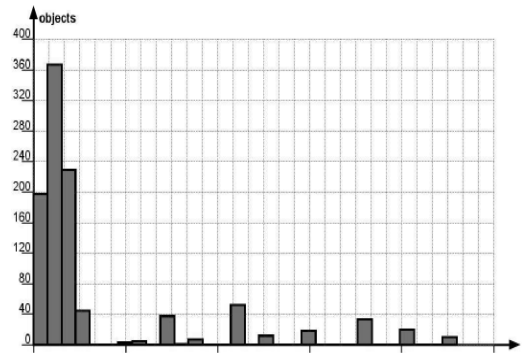

(4) Thickness

Figure 2. Distribution histograms of attributes

A particular point has been noticed at this stage; the values related to the straightening work realised by sub-contractors are not reliable since those workloads correspond to estimated times and not times of strictly achieved work. Unfortunately this case concerns more than two third of the records and decreases thus the quantity of exploitable data.
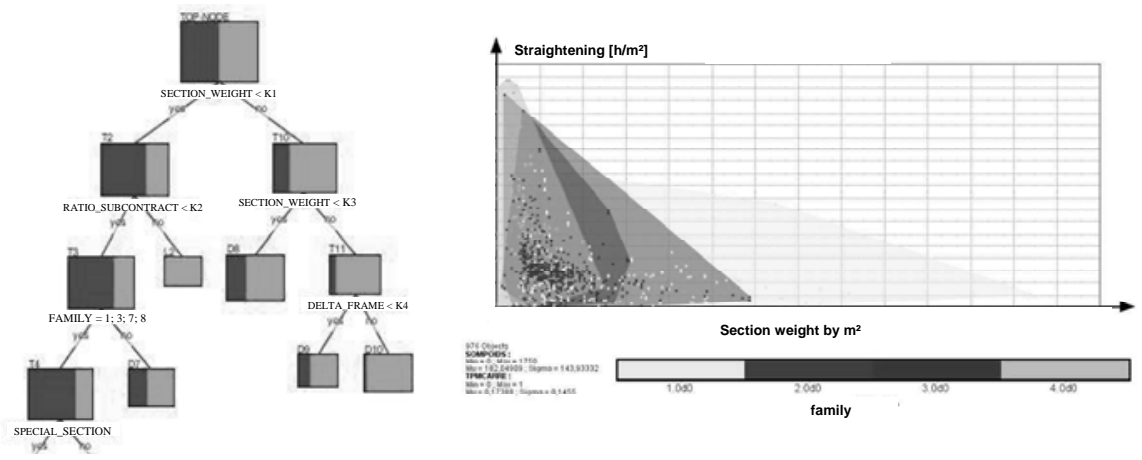

Figure 3. Statistical tools: (1) decision tree, (2) conditioned dots clouds

\subsection{Data exploration stage}

This work stage consisted in using different approaches to visualize the correlations existing between the attributes and the straightening workload in order to finally select the parameters having the most relevant influence on the straightening assessment. In order to fulfil this stage, four different approaches were used: a linear correlation analysis through dendrograms development, conditioned histograms, conventional dots clouds diagrams and decision trees analyses.

\subsection{Establishment of the formula}


This stage consisted in building the relation between the straightening cost [hour $\left./ \mathrm{m}^{2}\right]$ and the sections characteristics. The technique selected was the Artificial Neural Networks (ANN) method which is a powerful technique permitting to establish non linear relations (i.e. hyperbolic tangents) linking several inputs to a unique output.

The input attributes selected to generate the formula were the following ones: thickness, longitudinal stiffeners spacing, transversal girders spacing, ratio stiffeners spacing/girders spacing, section family, section weight $/ \mathrm{m}^{2}$, section weight/section length. After having chosen the input parameters, it was necessary to restrict the number of records in order to ignore the sections carrying disruptive information. In this optic, we have ignored the sections whose straightening work was done by subcontractors because time measurements of straightening were less reliable.

The formula was built exploiting 273 records and the correlation between the real value of straightening cost and the value estimated by the formula was 0,838 .
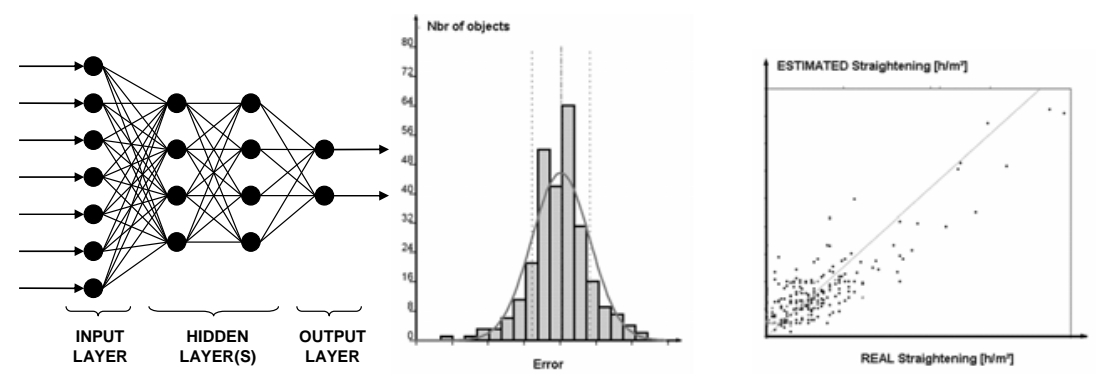

Figure 4 : errors diagrams relating to straightening work estimation (from PEPITo ${ }^{\circledR}$ )

\subsection{Limitation of the formula}

We have to notice that the generated formula has a limit. Firstly, since the recordings were restricted to the works realised by the shipyard workers, the quantity of data exploited was small and thus the robustness of the formula was not excellent. Moreover, when we have constructed the error diagrams, we have voluntarily tested the equation on the same data set than the one used to establish the relation. A consequence of this choice is that the precision given is not representative. Those precisions are optimistic in comparison to the precision obtained when the test set is different than the learning set. 


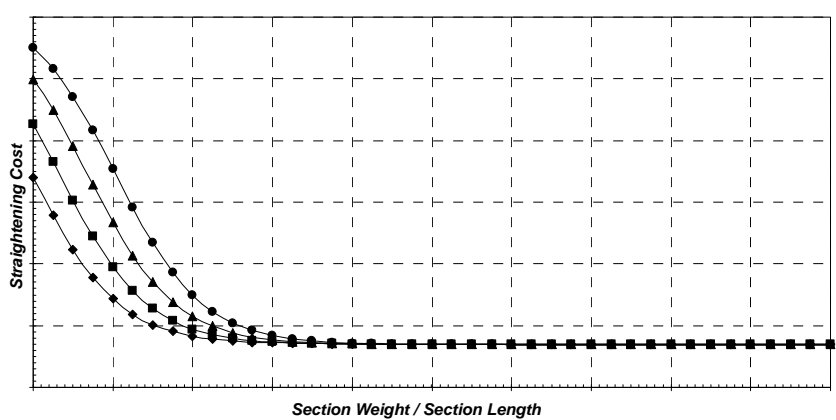

Figure 5 : decks straightening in function of the section weigth / section length parametered by the section family

\section{Distortions Data}

A measure campaign gathering the deck distortions of one passenger ship was realised recently at the Saint Nazaire shipyard. This brought new data and permitted to progress in the assessment of supplementary workloads caused by welding distortions. The distortions were measured after the blocks assembly stage and before the straightening operations. Each recorded value was related to the distortion occurring at the middle of rectangles (mesh division) delimited by consecutive longitudinal stiffeners and consecutive transversal girders. The database contained information about the scantling, the coordinates of the mesh division in the deck, the steel grade, neighbour structure element (bulkhead, longitudinal girder), sections characteristics (weight $/ \mathrm{m}^{2}$, fabrication workshop), etc. A particular attribute called "additional process" described the use of a distortion reduction technique (such as application of weld seams onto the plate between stiffeners). Indeed the initial scope of the measure campaign was to evaluate the impact of such distortions reduction techniques.

This database represents an interesting source of information and permitted to lead researches in two domains: firstly the distortions evaluation in function of scantling and secondly the analysis of distortions impact in the production.

\subsection{Distortions assessment}

The distortions assessment has followed the successive data mining stages investigate the correlation between attributes, extract meaningful parameters, fix exploitable data sub-sets and finally set up a formula through an ANN analysis.

Numerous attempts have been done to generate an effective estimation formula. It revealed that the exploitation of each distortion measure taken as unique record generates too much noise and involves thus a poor quality estimation (correlation around 0.5 for 23000 elements). By gathering the data per plate (1400 elements) the correlation increased to 0.7 and by gathering the data per section (108 elements) the correlation reached 0.9. This last solution was thus utilised to evaluate the welding deformations in absolute value. 

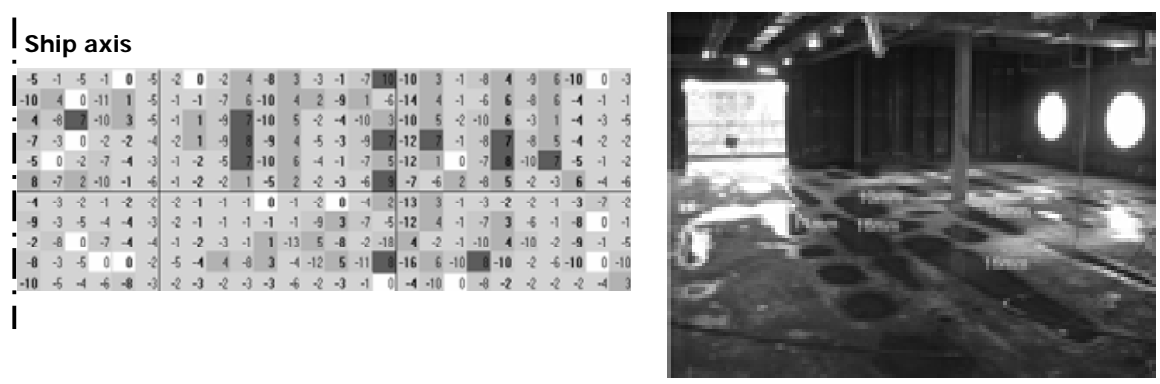

Figure 6 : (1) visualisation of a distortions field, (2) photo of a distorted deck

To generate the formula, the 108 sections concerned by the measured campaign were used and the following attributes were introduced as inputs: plate thickness, longitudinal stiffeners spacing, transversal girders spacing, ratio stiffeners spacing/girders spacing, section weight per $\mathrm{m}^{2}$, workshop of the section, additional process. The correlation between the real distortions and the values estimated by the formula was 0,947 (see Figure 7). This value is optimistic because the data set retained to test the formula was the same than the set selected to generate the formula.
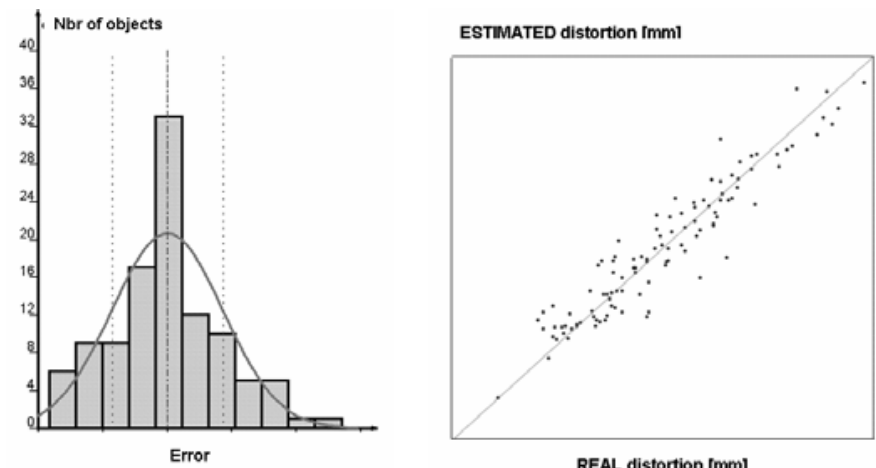

Figure 7 : errors diagrams relating to welding distortions estimation (from PEPITo ${ }^{\circledR}$ )

Here again, the formula has a limitation. The estimation is quite good inside the range values encountered by the attributes in the database but is not excellent outside. For instance, an estimation relating to a thickness lower than $5 \mathrm{~mm}$ or higher than $8 \mathrm{~mm}$ becomes rapidly approximate.

\section{Decks straightening in function of distortions}

This analyse corresponds exactly to the scope fixed before: assess the impact of welding distortions on the production. This analysis was realised by exploiting the database from the distortions measurement campaign. The idea was to generate a relation between the straightening workload and the level of welding deformations, with a more particular interest for the slope of the diagram curves than the precise estimation value of the straightening. 
Several attempts have been realised on dataset before obtaining relevant results. In the first attempt, each record of the database represented a rectangle (about $0,7 \mathrm{~m} * 2,5 \mathrm{~m}$ ) delimited by consecutive stiffeners and consecutive girders, the straightening workload associated was expressed in hour $/ \mathrm{m}^{2}$ and we have thus a precise value of distortion. The results given by the neural network technique were not good. In the second attempt, we gather the data by sections and thus each record of the database gave for a section: the average value distortions, the straightening workload expressed in hour $/ \mathrm{m}^{2}$ and the section characteristics. The results were once again not relevant.

Finally, we decided to continue to exploit the data by sections but we utilised the following attributes: total straightening workload on the section, sum of distortions on the section, average scantling (thickness, distance between stiffeners, and distance between girders). The results given by the neural network technique were quite good. Indeed the sensitivities of the generated formula (see Figure 8) show logic behaviour inside the validation domain:

- For a given thickness, the straightening increases when the distortions increase.

- For a given level of distortions, the straightening increases when the thickness increase. Indeed, it is necessary to heat longer a thick plate to reduce distortions.

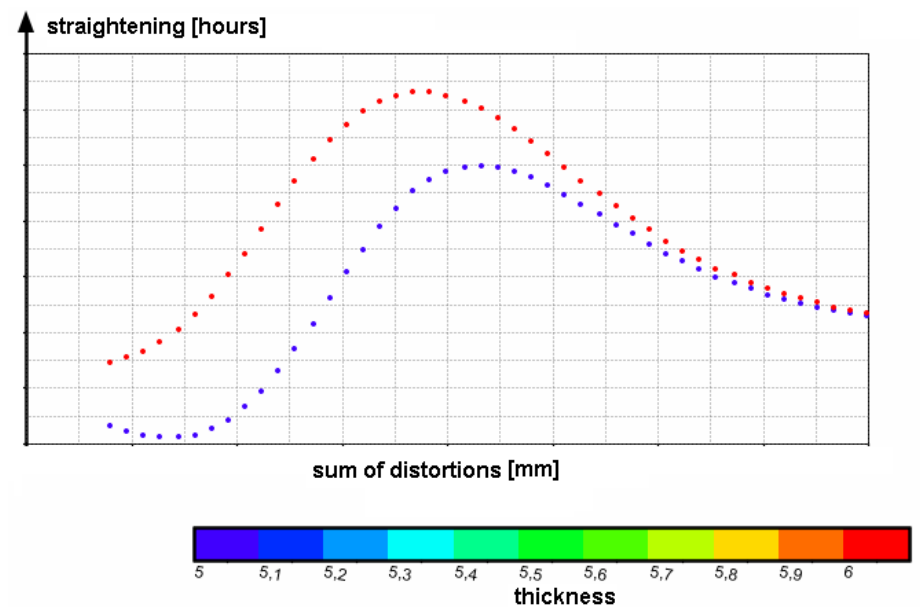

Figure 8 : decks straightening in function of the sum of distortions on a deck parametered by thickness

One of the difficulties encountered here to generate a reliable function is that few data were exploitable since the straightening of decks was often realised by subcontractors. The data of subcontractors corresponds to contract work times and not really executed work times.

\section{Steel working assessment}

The sections are generally constituted by a stiffened panel on which girders and bulkheads are welded. The steel working operations correspond to the adjusting and the fixing of those elements on panels in order to realise perfectly regular welds. The idea of this analyse was to correlate the increase of steel working operations in 
function of a distortions. Indeed more deformed are the plates, longer the workers will adjust steel elements to impose a precise contact between them.

The duration of steel works being highly dependant on section size, bulkheads number, etc. it was necessary to lead the analysis on similar sections and to introduce on top of preponderant attributes (distortions, thickness, distance between stiffeners, etc.) the section weight by $\mathrm{m}^{2}$ and also the bulkheads number divided by and the mesh divisions number.

This analysis seems satisfactory because the correlation between estimated value and real values of steel working is quite high and the sensitivities respect quite well the observations in situ.
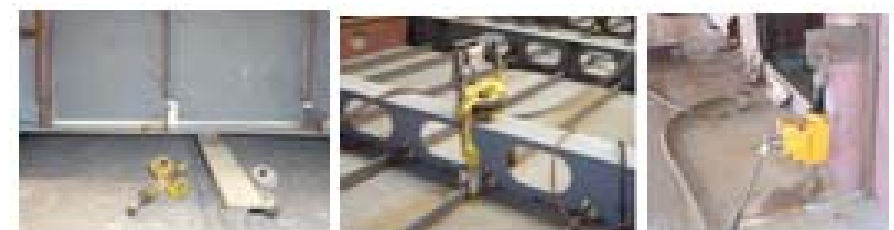

Figure 9 : tools for steel working operations

\section{Sections edges straightening assessment}

Similarly to the previous analysis, the sections edges straightening during their assembly to constitute block has been estimated. Only the distortions corresponding to the sections extremities have been introduced in the input "distortion" of the database. The results were not so perfect because few data about duration of those operations were exploitable.

\section{Economic analysis of techniques reducing the welding distortions}

At the Saint Nazaire shipyard, tests have been done to assess the effect of post welding techniques aiming to reduce the distortions. Those processes consist in welding, on the panel plates, different combinations of cords or stiffeners (see figure Figure 10) in order to generate counter deformations that could reduce the residual distortions appearing during fabrication.

An analysis has been realised in order to evaluate the economic profitability of those techniques taking into account the fact that they reduce the supplementary works of straightening, adjusting, etc.

The first step of this study was to assess the distortions reductions engendered by the techniques. We have here again exploited the data from the deformations measurement campaign to evaluate, for different scantling families, the distortions occurring with and without post welding processes. The distortions reductions were directly obtained by subtracting those last values. The Figure 10b presents the 
deformations reductions associated to each technique; a negative value means that the post welding process engenders higher distortions than the basic case, positive value means that the technique reduces efficiently the residual deformations.

The second step is to assess reparation (straightening and adjusting) costs avoided by the utilisation of post welding processes. We have exploited here the relations, detailed in previous chapters, between supplementary workloads and distortions. By introducing the distortions reductions in those formulas, we obtained for each process, the avoided reparation costs.

The third step is to compare the global earnings associated to the post welding processes in order to point out the best one. The earning is defined by the avoided costs of reparation (straightening and adjusting) minus the process cost. The Figure 10c presents the earnings associated to each technique; a positive value means that the technique is profitable since its utilisation cost engenders less straightening and adjusting costs.
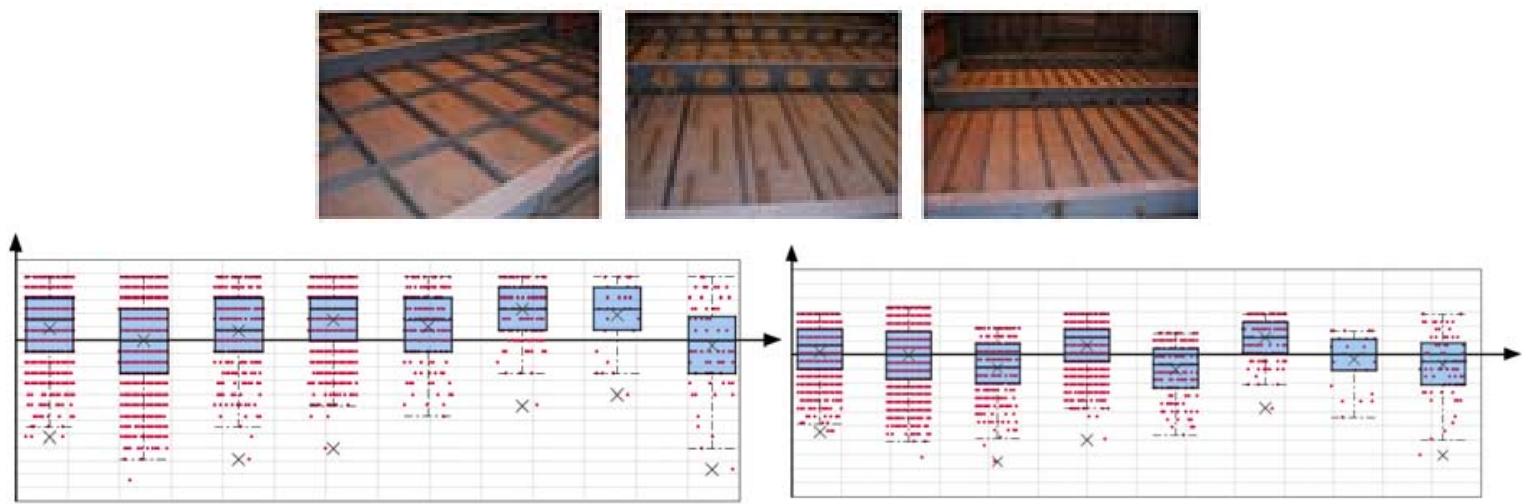

Figure 10 : (a) Post welding techniques to reduce deformations, (b) Deformations reduction, (c) Earnings associated to techniques

\section{Estimation of supplementary workloads in function of estimated distortions}

If we want to evaluate the supplementary workloads (straightening and steel working) for a ship for which the residual welding distortions are not known, a solution can be to introduce the deformations given by the formula detailed in chapter 3.1 as an input in the relations relative to straightening and steel working.

\section{Conclusions}

The thin plates that are more and more utilised in shipbuilding are strongly subjected to welding distortions. The supplementary operations to counter the relating problems of misalignment and lack of flatness involve non negligible over costs and delivery delays. It seems thus important to characterize their economic impact on the hull production.

This paper summarises several data mining analyses that exploited production data from the Saint Nazaire shipyard. A first study permitted, through a statistical 
analysis of 13 ships data, to establish a relation linking the straightening workload to the sections characteristics. Then, further to a distortions measurement campaign, the analyses have progressed; a formula was generated to estimate the distortions and others relations were established between the supplementary works (steel working, straightening of decks and sections edges) and the values of welding distortions. The generated formulas are useful to improve the research in the following domains: assessment of equipment profitability, production simulation, cost assessment of hull fabrication, structure optimization, design for production, etc.

Moreover the paper presents an economic comparison of post welding techniques aiming to reduce residual distortions. This analysis exploits the previously established relations.

\section{Acknowledgments}

The authors thank AKERYARD FRANCE (Saint-Nazaire) and Pepite (Liège), for the collaboration within sub-project II.1 of InterSHIP (Project $n^{\circ}$ TIP3-CT-2004506127 funded under the European Commission's Sixth Framework Programme) in which a part of the presented work has been realized. The authors thank also the European Marstruct project ( $\mathrm{n}^{\circ}$ TNE3-CT-2003-506141).

\section{References}

Losseau N., Caprace J.D., Archambeau D., Amrane A., Rigo P. (2008) Estimation of welding distortions and straightening workload through a data mining analysis DFE 2008

de Souza C., Tostes R. (2008) Shipbuilding Interim Product Identification and Classification System Based on Intelligent Analysis Tools COMPIT08, pp.481-493

Caprace JD., Losseau N., Archambeau D. (2007) A Data Mining Analysis Applied to a Straightening Process Database. In: International Conference COMPIT 2007 pp. 186-197.

KRUSZEWSKI J. (2007), Supervisory Neural Controller of the Ship Propulsion Plant, EAMARNET07

Bruce G. \& Morgan G. (2006) Artificial Neuronal Networks - Application to freight rates. In: International Conference COMPIT 2006, pp. 146-154

RODDY R. F., HESS D. E., FALLER W. E. (2006), Neural Network Predictions of the 4Quadrant Wageningen B-Screw Series, COMPIT06, pp.315-335 